\title{
In the Face of Negative Data, the Effects of Goal Type and Feedback Type on the Willingness to Continue to Participate Quantified-Self
}

\author{
Xiaofan Yang, Ding Pan \\ School of Management, Jinan University, Guangzhou, China \\ Email: yangxiaofan1996@163.com
}

How to cite this paper: Yang, X. F., \& Pan, D. (2020). In the Face of Negative Data, the Effects of Goal Type and Feedback Type on the Willingness to Continue to Participate Quantified-Self. American Journal of Industrial and Business Management, 10, 327-343.

https://doi.org/10.4236/ajibm.2020.102021

Received: January 7, 2020

Accepted: February 10, 2020

Published: February 13, 2020

Copyright $\odot 2020$ by author(s) and Scientific Research Publishing Inc. This work is licensed under the Creative Commons Attribution International License (CC BY 4.0).

http://creativecommons.org/licenses/by/4.0/

\begin{abstract}
In order to ensure the continuous participation of consumers in quantified-self, this paper studies the influence of target type and feedback type on the intention of quantified-self continuous participation in the face of negative data. The interaction between quantified-self goals and feedback types was taken as an independent variable, and self-efficacy was introduced as an intermediary to explain the influence of independent variables on the quantified willingness to continue to participate. In this study, the experimental method was used to prove the research hypothesis through two experiments. The results showed that in the face of negative data, when the type of quantified-self goal was promotion, compared with the task feedback, the adoption of ability feedback would lead to a higher willingness of quantified-self involvement due to a higher sense of self-efficacy. When the quantized-self goal type is prevention, compared with the task feedback, the adoption of capability feedback results in a lower willingness of quantified-self involvement due to a lower sense of self-efficacy. The results of relevant studies have positive guiding significance for enterprises to improve design methods to promote consumers' willingness to participate in quantified-self sustainability.
\end{abstract}

\section{Keywords}

Quantified-Self, Target Type, Feedback Type, Self-Efficacy, Continuous Willingness of Participation

\section{Introduction}

The age of the quantified-self has arrived. With the development of big data and Internet of things technology, consumers have more behavioral information than ever before (Azar, 2014; Lazer et al., 2009; Topol, 2013). Swan (2009) de- 
fined quantified-self as the process of consumers' tracking of information, such as self-physiology, behavior and environment. It is a process in which consumers track individual activities or lifestyle and relevant situational information they participate in (Guo, 2016). For consumers, quantified-self is helpful to enhance consumers' accurate control over themselves (Li, 2010; Ruckenstein, 2014), making consumer behavior more rational (Lupton, 2014), and driving the transformation of consumer behavior (Miltenberger, 2011). And consumers must insist on participating in quantified-self if they want to enjoy the benefits brought by quantified-self. Surveys have found that $34 \%$ of people who buy smart wearables take them off within six months, and up to $40 \%$ of fitness tracking consumers stop using them after a while. For enterprises, consumers' use of quantified-self devices for a long time will produce utility (Ledger \& McCaffrey, 2014; Guo, 2016) to improve the satisfaction of the product. Therefore, it is an important issue to improve the long-term participation willingness of consumers' quantified-self.

The mere presentation of quantified data makes consumers realize that their own status is not enough to drive their long-term participation in quantized-self (Guo, 2016). At present, many enterprises update quantitative tools and focus on providing social network services to consumers, hoping that users can improve their willingness to participate in the process of getting feedback and suggestions from community members and comparing results with community members. In addition, reminder service is also widely adopted by enterprises. The quantization tool triggers the reminder service according to the quantization activity goal, degree and time threshold set by consumers, so as to drive consumers to continuously participate in the quantization ego (Guo, 2016). However, these efforts of enterprises have not achieved significant results, and even caused consumer conflicts due to inappropriate reminder services (Van Berkel et al., 2015).

Consumers' participation in quantified-self is usually related to goals and feedback, based on which scholars have studied its influence on the intention of quantified-self to participate continuously (Li \& Zhang, 2018a). Consumers often have a purpose in using quantized-selfproducts. Li \& Zhang (2018b) divided quantified-self into promotion type and prevention type according to the regulation orientation theory. Individuals who set promotional goals wanted to accomplish self-improvement, while individuals who set preventive goals wanted to accomplish self-monitoring. The same quantized product and the same function may have different purposes. For example, individuals who set promotional goals use sports products to lose weight and become more beautiful, while individuals who set preventive goals want to lose weight. Different goals will give different responses to negative data (Woltin \& Yzerbyt, 2015). Feedback provides a summary of the implementation of the goals. Feedback can be divided into capability feedback and task feedback according to the target object. Some studies have found that task feedback can focus on specific tasks and promote the improvement of performance. However, other studies have found that strong availability of feedback (Ling et al., 2015) and support for feedback seeking can 
promote the satisfaction of individual psychological needs. In this case, the use of ability feedback can improve work performance (Dahling et al., 2015), while in the opposite case, the use of task feedback can reduce work performance (London, 2015). At present, no scholars have studied the effects of different goal settings and different feedback types on the continuous participation in quantification in the face of negative data. Secondly, on this basis, we study the psychological mechanism behind it.

\section{Theoretical Foundations}

The purpose of this chapter is to review and summarize the theoretical and empirical studies closely related to this study, and deduce the influence and mechanism of target type and feedback type on consumers' intention to use quantified-self continuously in the process of quantifying self. Firstly, the definition of the quantized self is sorted out and the focus on negative data is explained. Then the classification of quantified-self and the classification of feedback are sorted out, and then the related researches on mediation-self-efficacy are sorted out, and the relevant theories on the willingness to continue participation are sorted out to further demonstrate the influence of self-efficacy on the willingness to continue participation. Finally, the hypothesis of this paper are deduced based on the previous studies and theories.

\subsection{Quantified-Self}

Wolf and Kelly, editor-in-chief of wired, first proposed the concept of quantified-self in 2007, and Swan (2009) defined quantified-self as the process of consumers' tracking information about their physiology, behavior and environment. It is a process in which consumers track individual activities or lifestyle and relevant situational information they participate in (Guo, 2016).

The quantized-self is more authoritative than non-quantized feedback due to the data detail, and the data presented in the form of specific data rather than general presentation is considered to be more substantial and credible. Therefore, the results of quantified-self data will bring deeper impact to consumers, including more reflective thinking and thoughtful analysis (Li, 2010). In the process of quantifying-self, consumers involved in quantifying self may receive positive or negative measurement results with the help of tracking measurement tools (Baumgart, 2016). Positive data associated with the possibility of consumers to participate in the activities actively, and negative data associated with the possibility of consumers to participate in the activities negatively, but negative impact brought by the negative data is more effective than positive influence brought by the positive data (Algesheimer et al., 2010), that is to say, to participate in the quantified-self when consumers see negative data, with the positive data compared to bring more to participate, there will be a lower willingness to participate in the activities of quantified-self. At the same time, some scholars have studied that in the game of participation and negative feedback, negative feedback will reduce the amount of individual participation in activities. And as 
time goes on, the possibility of individuals participating in activities decreases (Dindo \& Tuinstra, 2010).

Therefore, we believe that negative data focusing on quantified-self will be more meaningful than positive data when studying the factors that influence consumers' continued participation in quantified-self behavior.

\subsection{Quantified-Self Classification}

There are many categories of quantified-self. Most scholars classify the quantized-self from the perspective of individual and community based on the consumer health situation. Li \& Zhang (2012) divided quantized-self into promotion type and prevention type according to different consumer goals and according to the regulation orientation theory. According to the type of needs served, the theory of regulatory orientations distinguishes two different types of regulatory orientations-promotional orientations related to advancement needs (advancement, growth, development, and cultivation, etc.) and preventive orientations related to security needs (security, protection, protection from harm, etc.). In contrast, promotional goals focus on gain, and growth, while preventive goals focus on avoidance, prevention, and error. Individuals who set promotional goals wanted to achieve self-improvement, such as exercising to lose weight and become more beautiful, while individuals who set preventive goals wanted to achieve self-monitoring, such as controlling their diet and not gaining weight. Individuals who promote orientation also set preventive targets. Individuals with promotional goals focus more on positive outcomes in the pursuit of goals and experience more emotions associated with joyfulness and depression. On the other hand, preventative target individuals pay more attention to the negative results in the pursuit of goals and experience more emotions related to relaxation and anger (Woltin \& Yzerbyt, 2015). At the same time, individuals who promote oriented-dominant state are more sensitive to information related to gains, while individuals who prevent oriented-dominant state are more sensitive to information related to losses.

\subsection{Feedback Type}

Feedback is individual goal setting and achievement of target (performance) of the reaction between a dynamic process, is also commonly used in organizational incentive strategy and behavior modification tools (Ilgen et al., 1979). Goal is the standard of individual evaluation of their performance, feedback is telling people how to meet these standards.

Feedback can be divided into positive feedback and negative feedback according to the titer. Although most studies have found that positive feedback helps improve job performance, DeNisi and Kluger (2000) have found that inaccurate positive feedback can actually mislead feedback recipients, increasing job errors and thereby reducing job performance. Negative feedback is not conducive to individual performance (Steelman et al., 2004). However, when negative feedback is communicated in an informational rather than controlling 
manner and the feedback source is highly credible (London, 2015), negative feedback can actually help improve individual performance. Domestic studies have also shown that positive feedback is not always more effective than negative feedback in promoting the improvement of performance, but also depends on the content of feedback (Zhang \& Li, 2018), feedback style (Li, 2012), and feedback source characteristics (Li, 2010).

Feedback can be divided into capability feedback and task feedback according to the target object. Task feedback is targeted at the task itself, which is compared with some standard of the task, so that individuals can focus on the gap between the task itself and the standard, instead of making social comparison. Capability feedback is targeted at individual competence and is carried out through social comparison. Studies have found that capability feedback can hinder the improvement of performance, while task feedback can focus on specific tasks and promote the improvement of performance. However, other studies have found that strong availability of feedback (Ling et al., 2015) and support for feedback seeking can promote the satisfaction of individual psychological needs. In this case, the adoption of capability feedback can improve work performance (Dahling et al., 2015), while in the opposite case, the use of task feedback can reduce work performance (London, 2015).

\subsection{Self-Efficacy}

"Self-efficacy" was first proposed by Bandura (1977). It refers to "the belief, judgment or self-grasp and feeling of the subject before the individual is going to do something". It is one of the individual ability to oneself consciousness, which can make oneself to achieve a specific purpose. The consciousness will impact thinking, mood, motivation and behavior of individual, such as adjusting individual's choice of behavior, the size of the commitment, and decided to its ability to exhibit in the special task (Yao, 2008). Bandura conducted a lot of research and experiments and found that self-efficacy mainly comes from four channels: 1) Direct experience, also known as past success or failure experience, refers to an individual's perception of the level of achievement in actual activities. 2) Indirect learning, also known as the demonstration effect, refers to the observation and comparison of other people's activity experiences and their achievement levels. In other words, observing others' efforts to achieve success will also increase their sense of self-efficacy. The higher the similarity between the observed objects and themselves and the higher the relevance of completing the task, the better the effect of this sense of self-efficacy. 3) Verbal persuasion refers to the verbal encouragement given by others to an individual to complete a certain task. When individuals receive such signals, they tend to make greater efforts and persist longer. This signal becomes even more significant when the individual is completing the task and entering a bottleneck period. 4) Emotional and physical arousal, which refers to an individual's physical and mental response to a situation or task. Bandura believed that people's perception of their own abilities would be based on their current physiological and emotional states. Positive 
emotions have been proved to be conducive to the promotion of self-efficacy, while negative emotions often show inhibitory effects (Bandura, 1977).

Self-efficacy can affect four aspects of an individual: behavioral choice, motivational effort, cognitive process and emotional process. 1) Behavioral choice. Individuals with a high sense of self-efficacy will actively respond to tasks higher than their own ability level, regard difficulties as challenges and develop their skills. In contrast, individuals with a low sense of self-efficacy respond to tasks above their level of competence with passive avoidance and put themselves on the defensive to avoid threats. 2) Motivational efforts. Individuals with a high sense of self-efficacy are more motivated to complete tasks and are more willing to invest, pay and persist, whereas individuals with a low sense of self-efficacy are less willing to invest and persis. 3) Thinking process. Individuals with a high sense of self-efficacy will focus on solving problems, and when they encounter difficulties and setbacks, they will conceive successful scenarios to motivate them to continue their efforts. Individuals with low self-efficacy, on the other hand, visualise failure. 4) Emotional process. When facing negative situations with uncertain factors such as pressure, difficulty and misfortune, self-efficacy will dominate individual's stress function, such as activating individual's emotional expression such as anxiety. Individuals with low self-efficacy will produce a series of emotional reactions such as anxiety and depression in order to fail to cope with the threat, and take protective defensive behaviors to deal with the threat. These behaviors not only hinder the development of individual personality, but also inhibit the functional play of the subject in the environment.

In addition to its effect on individual psychology, self-efficacy has been increasingly studied in management and marketing. As a dependent variable, a large number of studies have confirmed the direct effect of self-efficacy on individual behavior. Zhang \& $\mathrm{Li}$ (2015) proved that compared with individuals with low self-efficacy, individuals with high self-efficacy are more willing to choose performance compensation rather than fixed compensation in work. Ge Zhu et al. (2010) took the mobile auction business of eBay and Taobao as the research object and confirmed that consumers' self-efficacy would have a direct impact on their brand attitude and purchase intention.

On the other hand, self-efficacy is also focused as an intermediary variable between the soft environment and individual behavior performance (Meng et al., 2010). The setting of enterprise marketing environment will influence consumers' self-efficacy and consumer behavior. Chi et al. (2012) proposed that when consumers receive a service, their interpretation of their role in the service will affect their sense of self-efficacy and the completion of the service.

\subsection{Willingness to Continue to Use}

In 1980, Oliver first proposed the Expectation Confirmation Theory (ECT) in his research. The theoretical model consists of five variables, which included perceived performance, expectations, experience gaps, satisfaction and willingness to continue to buy. Later, ETC was widely used in theoretical research on 
the continued use of users, with scholars learning from online learning (Cheng, 2014; Zhang et al., 2016), mobile applications (Yin \& Li, 2017) and knowledge sharing (Luo \& Li, 2017) and other different research areas confirm the applicability of the theory of expectation confirmation.

In the follow-up study, based on the integration of the ECT model, some scholars have suggested the model of continuous use of information system, which named as the Expected Confirmation Model of IS Continuance (ECM-ISC). The model consists of four core variables: perceived usefulness, satisfaction, expectation recognition, and willingness to continue to use.

Scholars extends the dependent variable from the original willingness to continue to use to continuous use behavior on the basis of ECM-ISC. Subsequently, Bhattacherjee \& Perols (2008) expanded the initial escalate model of The ECM-ISC on the basis of this study, introducing variables such as IT self-efficacy and Facilitating conditions. Remove the direct effects of perceived usefulness on satisfaction, resulting in an extended theoretical model of continuous use (EECM). EECM is a better place to reveal the individual differences of the user and the impact of external environmental factors on the willingness and behavior of continuous use.

Based on the EECM, this paper once again verifies the positive effect of self-efficacy on the willingness to continue to use.

\subsection{The Impact of Quantified-Self Target Types and Feedback Types on Consumers' Willingness to Quantified-Self Sustained Participation}

This paper divided the quantified-self into the promotion and prevention type according to the goal. In quantified-self situations, consumers of promotional targets are relatively less sensitive, have less negative emotions, and have no particularly negative expectations about the future when faced with negative data results. Consumers with preventive targets are more sensitive, have more negative emotions, and are more likely to have negative expectations and give up. Furthermore, consumers of both target types are somewhat less ensamounting the idea of quantifying their willingness to engage on a sustainable scale, but consumers with preventive targets are more likely to have negative expectations and abandoned ideas."

This article distinguishes feedback into capability feedback and task feedback. Task feedback is aimed at the task itself, through the task of a certain standard comparison, so that the individual pay attention to the gap between the task itself and the standard, without social comparison; Capability feedback is directed at individual abilities, conducted through social comparisons. Facing negative data can be seen as an uptrend social comparison. In the face of upward social comparison, there are two opposite results: the contrast effect and the assimilation effect. The contrast effect causes individuals to lower self-evaluation and take action away from the phenomenon. The assimilation effect will give the individual a stronger level of self-improvement and self-confidence. The reason for 
these two results is the similarity of individuals to comparison objects. The assimilation effect of the upward comparison will lead the individual to have a more positive attitude in the future, resulting in a more positive emotional experience.

In a device that quantified-self, individuals are unable to know the information about the object they compare with themselves, so that they cannot perceive the significant differences between themselves and other comparison objects, and tend to think that they are similar to the object of comparison. Therefore, the use of capacity feedback is more likely to occur assimilation effects. That is, in the face of negative data results, there is a stronger level of self-improvement and self-confidence. In the pursuit of goals, can be seen as raising the intrinsic motivation. The task feedback focuses on the task itself, allowing the individual to focus on the distance between the current task completion and the "standard", and the consumer can more accurately know where they are lacking and the direction of future efforts than the ability feedback.

In the case of the promotion-oriented goal of the consumer, when the type of feedback is the capability feedback, the consumer's goal of improving themselves coincides with the desire to improve themselves, produces assimilation effects, has higher self-confidence, and therefore produces a higher willingness to participate continuously. When the type of feedback is task feedback, the consumer is accurately informed of the gap between themselves and expectations, and the consumer is now more sensitive to earnings, and this gap is a loss. Therefore, there will be no greater willingness to continue to participate.

In the case of a consumer's goal as a preventive target, when the type of feedback is capability feedback, the consumer's goal of avoiding loss does not match the desire to improve itself, does not have an assimilation effect, and therefore does not produce a higher willingness to participate continuously. When the type of feedback is task feedback, the accuracy of the task feedback coincides with the consumer's emphasis on caution and accuracy, resulting in a higher willingness to participate continuously.

Thus, we propose the following assumptions:

H1a: When the type of target is promotion, using capability feedback leads to a higher willingness to quantified-self continuous participation than task feedback.

H1b: When the type of target is prevention, using capability feedback leads to a lower willingness to quantified-self continuous participation than task feedback.

\subsection{The Intermediary Effect of Self-Efficacy}

Bandura believed that people's perception of their own abilities would be based on their current physiological and emotional states. Positive emotions have been proved to be conducive to the promotion of self-efficacy, while negative emotions often show inhibitory effects (Bandura, 1977).

When faced with negative data results, consumers of both promotional and 
preventive goals will generate negative emotions, which will lead to a low sense of self-efficacy, thus reducing the willingness to continue to participate.

In the case that the target of consumers is the promotion, when the type of feedback is the capability feedback, the goal of self-improvement of consumers is consistent with the intention of self-improvement, resulting in assimilation effect and more positive emotions. Therefore, the sense of self-efficacy will be improved, resulting in a higher willingness to continue to participate. When the feedback type is task feedback, consumers are accurately informed of the gap between themselves and expectations, which is a kind of loss. At this time, consumers are more sensitive to the benefits, so they cannot generate more positive emotions, so they will not generate higher willingness to continue to participate.

When the target of consumers is preventive, and the type of feedback is capability feedback, the goal of consumers to avoid loss is not consistent with their desire to improve themselves, and they cannot produce assimilation effect or positive emotion, so they will not have a higher willingness to continue to participate. The accuracy of task feedback is consistent with consumers' emphasis on caution and accuracy. Meanwhile, it also provides detailed solutions for the completion of the target.

Individuals with a high sense of self-efficacy respond positively to tasks above their level of competence, seeing difficulties as challenges and developing their skills. In contrast, individuals with a low sense of self-efficacy respond to tasks above their level of competence with passive avoidance and put themselves on the defensive to avoid threats. In terms of motivational efforts, individuals with high self-efficacy are more motivated to complete tasks and are more willing to invest, pay and persist, whereas individuals with low self-efficacy are less willing to invest and persist. At the same time, according to the EECM, a high sense of self-efficacy leads to a high willingness to continue participation, while a low sense of self-efficacy leads to a low willingness to continue participation (Bhattacherjee \& Perols, 2008).

Therefore, we propose the following hypothesis:

H2: Self-efficacy plays a mediating role in goal type and feedback type on consumers' intention of quantified-self continuous participation.

H2a: when the quantified-self goal type is promotion, compared with task feedback, the adoption of capability feedback will lead to a higher sense of self-efficacy, thus showing a higher willingness to participate in quantified-self sustainability.

$\mathrm{H} 2 \mathrm{~b}$ : when the quantified-self goal type is prevention, compared with task feedback, the adoption of capability feedback will lead to a lower sense of self-efficacy and thus a lower willingness to participate in the quantified self-continuation.

\section{Theoretical Model}

From the perspective of individuals, this paper studies the formation mechanism 
of quantified-self goal type and feedback type acting together on the intention of quantified-self continuous participation in the presence of negative data, and explains the principle of this mechanism through the mediation of self-efficacy. The independent variables are the target type and the feedback type, the interaction between the two (Capability ${ }^{\star}$ Promotion; Task ${ }^{\star}$ Prevention) have a positive effect on willingness to stay involved. Self-efficacy plays a completely mediating role, and the independent variable improves the willingness to participate continuously by enhancing the self-efficacy of individuals in Figure 1.

\section{Experimental Research and Hypothesis Testing}

Two experiments were carried out in this study. Experiment 1 quantified the motion data, manipulated the goal type and feedback type, and tested the interaction (main effect) of quantized self goal type and feedback type on consumers' willingness to continue to participate in quantified-self. Experiment 2 quantified reading ability, manipulated negative results, quantified-self goal types and feedback types again, and verified the mediating effect of main effect and self-efficacy.

\subsection{Experiment 1}

There were 120 subjects in Experiment 1, and each of them was paid for the experiment. After excluding 4 invalid questionnaires (positive perception of the data), a total of 116 valid questionnaires were obtained in Experiment 1, including 31 males and 85 females, aged between 18 and 40. According to the test, gender has no significant influence on consumers' willingness to participate in quantified self-sustainability $(\mathrm{F}(1,114)=0.432, p>0.05)$, and age has no significant influence on consumers' willingness to participate in quantified self-sustainability $(\mathrm{F}(1,114)=0.376, p>0.05)$, so it was not taken into consideration in the subsequent statistical analysis.

\subsubsection{The Experimental Process}

Firstly, subjects were asked to read experimental materials of different target types and complete the manipulation scale for target types. In the case of promotional goals, words such as pursuit, success, and achievement appear. In the case of preventive targets, words such as avoidance, prevention, error, etc.

The specific description of the promotional goal is as follows:

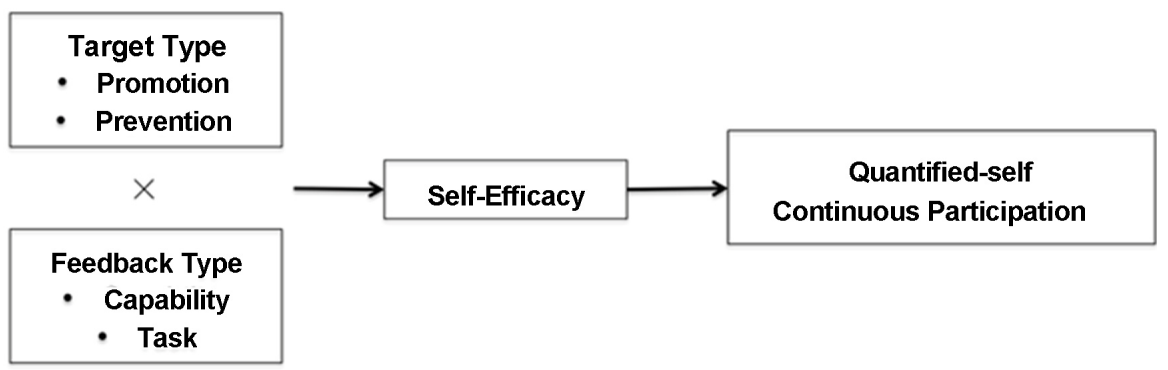

Figure 1. Theoretical model. 
Please imagine the following scene: In modern society, busy is the main melody, the long-term sports lead to obesity. Therefore, you are using a sports product recently, which can use modern technology to monitor movement data in real time, including movement track, time, basic metabolism and consumption monitoring. The measurement movement data, raises the outstanding movement ability, can help you to shape the perfect figure, the good appearance condition. From the long-term use of this product, you can bring great benefits in the future, harvest unexpected results.

The specific description of preventive objectives is as follows:

Please imagine the following scene: in modern society, busy is the main melody, long time sports lead to physical function decline. Imagine you are currently using a sports product that USES modern technology to monitor movement data in real time, including movement trajectory, time, basal metabolism, and consumption. Do not measure motion data, monitor each function of the body hard with intuitive data, bring about weak system, make you appear easily body fat, perennial insomnia and the state of sub-health. Long-term use of this product can reduce or eliminate the money used to treat diseases of the body, and avoid regret and regret in the future.

Then, the subjects were informed that the next step was a formal experiment. The subjects were presented with a paragraph of text and pictures of different types of feedback, and were asked to answer three questions related to the picture and complete the manipulation scale of feedback types. In the capability feedback situation, participants were told that their test results exceeded the percentage of the participants. In the case of task feedback, participants were told how their test results compared to the norm for the normal population. The contents are as follows:

The experimental materials of ability feedback are described as follows: "You ran 3.9\% faster than they did today and burned $4.2 \%$ more calories than they did today."

The experimental materials of task feedback are as follows: "You ran $2 \mathrm{~km}$, spend 12 minutes per $\mathrm{km}$ (Experts say $5-7 \mathrm{~min} / \mathrm{km}$ is effective), and you burn 48 calories. (Experts say burning 90 calories is effective.)"

For the purpose of disguising the experiment, the subjects were required to complete the design score of the sports product interface.

Finally, the subjects were asked to fill in the willingness scale for continuous use and demographic information, including gender, age, occupational status.

\subsubsection{The Experimental Results}

The manipulation of the target type in this experiment was successful $(\mathrm{F}(1,112)$ $=19.030, p<0.05)$ ). The manipulation of feedback type in this experiment is successful $(\mathrm{F}(1,112)=33.773, p<0.05)$.

Objective type and feedback type were used as independent variables, and participants' willingness to participate was used as dependent variable for the analysis of two-factor variance. The main effect of the target type was not signif- 
icant $(\mathrm{F}(1,112)=0.326, p=0.569>0.1)$, and the main effect of feedback type was not significant. $((\mathrm{F}(1,112)=0.184, p=0.669>0.1)$, the interaction between target type and feedback type was significant $(\mathrm{F}(1,112)=12.521, p=0.00<$ 0.05).

Then we did a simple effect analysis. Under the promotional target, the results showed that the willingness of continuous participation in the capability feedback group was significantly higher than that in the task feedback group $\left(\mathrm{M}_{\text {capabi- }}\right.$ ity $=5.59, \mathrm{SD}=1.163 ; \mathrm{M}_{\text {task }}=4.68, \mathrm{SD}=1.137, \mathrm{~F}(1,192)=10.836, p=0.002<$ $0.05)$. Under the preventive target, the continuous participation intention of the capability feedback group was significantly lower than that of the task feedback group $\left(\mathrm{M}_{\text {capability }}=4.76, \mathrm{SD}=0.83 ; \mathrm{M}_{\text {task }}=5.79, \mathrm{SD}=1.069, \mathrm{~F}(1,192)=21.743, p\right.$ $=0.00<0.05)$, which verified hypothesis H1a, H1b.

\subsection{Experiment 2}

There were 200 subjects in Experiment 2, and each of them was paid for the experiment. After eliminating 6 invalid subjects, a total of 194 valid subjects were obtained in Experiment 2, including 60 males and 134 females, aged between 18 and 40. According to the test, gender has no significant influence on consumers' willingness to participate in quantified-self sustainability $(\mathrm{F}(1,192)=0.302, p>$ $0.05)$, and age has no significant influence on consumers' willingness to participate in quantified-self sustainability $(\mathrm{F}(1,192)=2.987, p>0.05)$, so it is not taken into account in the subsequent statistical analysis.

\subsubsection{The Experimental Process}

Firstly, the subjects were told to test their concentration. They should read experimental materials of different target types, and complete the manipulation scale of target types.

In Experiment 2, reading ability was taken as the background, in which the specific description of the promotion goal was as follows:

As long as you have the ability to read, everything can be easily solved. By testing the relevant standards of reading ability, we can reflect our reading speed and understanding of reading content, and cultivate our reading ability better than others, so as to obtain more information in the same time and successfully shape stronger logical thinking.

The specific description of preventive objectives is as follows:

Without the ability to read, anything can get in the way. By testing the relevant standards of reading ability, we can reflect our reading speed and understanding of reading content, get rid of poor reading ability, so as to prevent the omission of important information and avoid the risk of chaotic logical thinking.

Then, the subjects were told that the next step was a formal experiment. The subjects were presented with an article unrelated to the experiment for 30 seconds, and then asked to answer seven questions related to the article. After that, subjects were showed experimental materials of different feedback types to the subjects, and complete the manipulation scale of feedback types. 
The experimental materials of capability feedback are described as follows: "Here are the results of your reading tests: You read faster than $28 \%$ and more accurately than $28 \%$ ".

The experimental materials of task feedback are as follows: "Here are the results of your reading ability test. your reading speed is approximately 323 words/min (the normal standard is 350 - $500 \mathrm{words} / \mathrm{min}$ ), and your reading accuracy is $3 / 7$."

Then the subjects were asked to fill in a scale of self-efficacy, willingness to use continuously., demographic information, including gender, age, occupational status, etc.

\subsubsection{The Experimental Results}

The manipulation of the target type is successful $(\mathrm{F}(1,192)=22.232, p=0.000<$ $0.001)$ ). The manipulation of feedback type in this experiment is successful ( $\mathrm{F}(1$, 192) $=10.755, p=0.001<0.05)$ ).

Objective type and feedback type were used as independent variables, and participants' willingness to participate was used as dependent variable for the analysis of two-factor variance. The main effect of the target type was not significant $(\mathrm{F}(1190)=0.428, p=0.514>0.05)$, The main effect of feedback type was not significant $((\mathrm{F}(1190)=0.490, p=0.485>0.1)$, The interaction between target type and feedback type was significant $(\mathrm{F}(1190)=9.082, p=0.003<0.01)$.

Then we did a simple effect analysis. Under the promotional target, the results showed that the willingness of continuous participation in the capability feedback group was significantly higher than that in the task feedback group (M ability $=5.97, \mathrm{SD}=0.763 ; \mathrm{M}$ task $=4.86, \mathrm{SD}=1.084, F(1,192)=25.83, p=0.00$ $<0.05)$. Under the preventive target, the continuous participation intention of the capability feedback group was significantly lower than that of the task feedback group ( $\mathrm{M}$ capability $=4.97, \mathrm{SD}=1.61 ; \mathrm{M}$ task $=5.68, \mathrm{SD}=0.976, \mathrm{~F}(1,192)$ $=4.753, p=0.033<0.05)$, which verified hypothesis H1a, H1b.

The mean value of the three questions of self-efficacy was used as the score of self-efficacy of the subjects, and the Bootstrap method (model 8) proposed by Hayes (2013) was used for mediating effect test (the number of repeated samples was set as 5000 and the confidence was $95 \%$ ).

Data analysis showed that the test results of the mediating effect of self-efficacy did not include 0 (LLCI $=0.1232$, ULCI $=1.2056)$. More specifically, low self-efficacy mediates the negative effect of feedback type on the willingness to continue participation under the promotion target $(\mathrm{LLCI}=-0.6798$, ULCI $=$ -0.1578 .), while high self-efficacy mediates the positive effect of feedback type on the willingness to continue participation under the defensive target (LLCI = 0.0727, ULCI $=0.8669$ ), which verified hypothesis H2a, H2b.

To sum up, this study uses the experimental method to prove the research hypothesis through two experiments. In the face of negative data, this paper makes a preliminary exploration on how the quantified-self target type and feedback type affect consumers' intention of quantified-self sustaining use, and 
investigates the mediating role of self-efficacy in the interaction effect of target type and feedback type on consumers' intention of quantified-self sustaining use.

\section{Research Conclusion and Prospect}

\subsection{Research Conclusions}

This study uses the experimental method to prove the research hypothesis through two experiments. In the face of negative data, this paper makes a preliminary exploration on how the quantified-self goal type and feedback type affect consumers' intention of quantifying-self sustaining use, and investigates the mediating role of self-efficacy. Specifically, our findings suggest that in the face of negative data, when the quantified-self goal type is promotional, the adoption of capacity feedback results in a higher willingness to quantize self-involvement due to a higher sense of self-efficacy. When the quantified-self goal type is preventive, the adoption of capability feedback results in a lower willingness to quantize self-involvement due to a lower sense of self-efficacy.

\subsection{Theoretical Contribution}

1) The research on quantified-self in the consumption field has been enriched. Existing researches on quantified-self focus on the human-computer interaction field, focusing on the tool design, mining ideas, key components and other aspects of quantified self. This paper focuses on the field of consumption, from the perspective of consumers to think about the quantified self related issues.

2) The formation mechanism of consumers' willingness to quantify their own continuous participation has been enriched. From the perspective of individuals, this paper studies the formation mechanism of quantified-self goal type and feedback type, which is a supplement to the research on quantified self-sustainability.

3) Enrich the research on self-efficacy. It expands the research of self-efficacy in marketing field and enriches the role of self-efficacy as an intermediary.

\subsection{Management Enlightenment}

1) For consumers, it helps them to understand the factors that can continuously participate in quantifying themselves, so as to adjust the ways of quantifying themselves, achieve the utility of quantifying themselves, and achieve rational and accurate control of themselves.

2) For relevant enterprises, a design method can be found to promote consumers' quantified willingness to participate in the continuous participation, so as to generate stickiness and higher satisfaction among consumers, thus bringing higher benefits to enterprises.

\subsection{Research Limitations and Future Prospects}

The limitations of this study are as followed: first of all, regarding the selection of experimental products, this paper directly determines the experimental products based on a small range of interviews before the experiment. It is possible to ignore the interference of other factors, and the experiment is still not rigorous. 
It is a question whether the conclusion can be generalized to the realistic quantified-self scenario. Secondly, limited by time, energy and resources, the object samples of this study are relatively homogeneous, which can further enrich the research samples in the future. The experimental subjects in this paper are mostly college students. However, individuals of different age groups, social classes and incomes may have different reactions to this problem. Therefore, this paper has certain limitations in the selection of subjects, and fails to cover all consumers comprehensively.

The related variables in this study can be further enriched. In the future research, instead of restricting specific population as subjects, different subjects will be randomly selected offline or online for simulation experiments, so as to make the experimental data more consistent with real life and more universal. In the future research, we can cooperate with some enterprises that produce quantized self-products, collect the real data of consumers in the background, and start the research from the real big data.

\section{Conflicts of Interest}

The authors declare no conflicts of interest regarding the publication of this paper.

\section{References}

Algesheimer, R., Borle, S., \& Dholakia, U. M. (2010). The Impact of Customer Community Participation on Customer Behaviors: An Empirical Investigation. Marketing Science, 29, 756-769. https://doi.org/10.1287/mksc.1090.0555

Azar, B. (2014). QnAs with Davis Masten and Peter Zandan. Proceedings of the National Academy of Sciences of the United States of America, 111, 1662-1663. https://doi.org/10.1073/pnas.1320462110

Bandura, A. (1977). Self-Efficacy: Toward a Unifying Theory of Behavioral Change. Advances in Behaviour Research and Therapy, 1, 139-161.

https://doi.org/10.1037/0033-295X.84.2.191

Baumgart. R. (2016). How Does Quantified Self Run? In Proceedings of the 22th Americas Conference on Information Systems (pp. 1-9). San Diego, CA: ACM.

Bhattacherjee, A., \& Perols, J. (2008). Information Technology Continuance: A Theoretic Extension and Empirical Test. Journal of Computer Information Systems, 49, 17-26. https://doi.org/10.1080/08874417.2008.11645302

Cheng, Y. M. (2014). What Drives Nurses 'Blended e-Learning Continuance Intention. Journal of Educational Technology and Society, 17, 203-215.

Chi, K. Y., Chan, K. W., \& Lam, S. S. K. (2012). Do Consumer and Employees Enjoy Services Participation? Synergistic Effects of Self- and Other-Efficacy. Journal of Marketing, 76, 121-140. https://doi.org/10.1509/jm.11.0205

Dahling, J., O’Malley, A. L., \& Chau, S. L. (2015). Effects of Feedback Motives on Inquiry and Performance. Journal of Managerial Psychology, 30, 199-215.

https://doi.org/10.1108/JMP-12-2012-0409

DeNisi, A. S., \& Kluger, A. N. (2000). Feedback Effectiveness: Can 360-Degree Appraisals Be Improved. Academy of Management Perspectives, 14, 129-139.

https://doi.org/10.5465/ame.2000.2909845 
Dindo, P., \& Tuinstra, J. (2010). A Class of Evolutionary Models for Participation Games with Negative Feedback. Computational Economics, 37, 267-300. https://doi.org/10.1007/s10614-011-9253-3

Guo, L. (2016). Quantified-Self 2.0: Using Context-A ware Services for Promoting Gradual Behaviour Change (pp. 1-18). Working Papers of Computers and Society.

Hayes, A. (2013). Introduction to Mediation, Moderation, and Conditional Process Analysis. Journal of Educational Measurement, 51, 335-337.

Ilgen, D. R., Fisher, C. D., \& Taylor, M. S. (1979). Consequences of Individual Feedback on Behavior in Organizations. Journal of Applied Psychology, 64, 349-371. https://doi.org/10.1037/0021-9010.64.4.349

Lazer, D., Alex, P., \& Lada, A. (2009). Life in the Network: The Coming Age of Computational Social Science. Science, 323, 721-723. https://doi.org/10.1126/science.1167742

Ledger, D., \& McCaffrey, D. (2014). Inside Wearables: How the Science of Human Behavior Change Offers the Secret to Long-Term Engagement (pp. 1-17). Endeavour Partners LLC.

Li, D. J., \& Zhang, Y. D. (2012). Quantified Self in the Field of Consumption: Research Review and Outlook. Foreign Economy and Management, 8, 3-17.

Li, D. J., \& Zhang, Y. D. (2018a). Effect of Quantified Self and Its Influence Mechanism on Consumer Participation. Science and Technology, 31, 112-124.

Li, D. J., \& Zhang, Y. D. (2018b). Why Consumers Give up: The Internal Mechanism of Quantifying the Formation of Self-Sustaining Participation Intention. Nankai Management Review, 21, 118-131.

Li, I. (2010). Personal Informatics and Context: Using Context to Reveal Factors that Affect Behavior. Journal of Ambient Intelligence and Smart Environments, 4, 71-72. https://doi.org/10.3233/AIS-2011-0130

Li, L. (2012). Leadership Feedback Titer, Feedback Style and Subordinate Regulation Focus: Discussion on Interactive Influence on Subordinate Creativity. Science of Science and Technology Management, No. 5, 150-159.

Ling, Y. L., Abdullah, A. G. K., \& Ismail, A. (2015). Feedback Environment and Coaching Communication in Malaysia Education Organizations. Asian Journal of Social Sciences \& Humanities, 4, 66-73.

London, M. (2015). The Power of Feedback: Giving, Seeking, and Using Feedback for Performance Improvement. New York: Routledge.

Luo, X. L., \& Li, M. (2017). A Preliminary Study on the Sharing and Use of Free Online Academic Resources-A Case Study of Baiduwenku. Modern Intelligence, 37, 100-105.

Lupton, D. (2014). Self-Tracking Modes: Reflexive Self-Monitoring and Data Practices. SSRN Electronic Journal, 391, 547-551. https://doi.org/10.2139/ssrn.2483549

Meng, H., Liang, Q. F., \& Shi, Y. Y. (2010). Goal Orientation and the Relationship between Self-Efficacy and Subjective Well-Being. Journal of Psychology, 33, 96-99.

Miltenberger, R. G. (2011). Behavior Modification: Principles and Procedures (5th ed.). Belmont, CA: Wadsworth Cengage Learning.

Oliver, R. L. (1980). A Cognitive Model for the Antecedents and Consequences of Satisfaction. Journal of Marketing Research, 17, 460-469.

https://doi.org/10.1177/002224378001700405

Ruckenstein, M. (2014). Visualized and Interacted Life: Personal Analytics and Engagements with Data Doubles. Societies, 4, 68-84.

Steelman, L. A., Levy, P. E., \& Snell, A. F. (2004). The Feedback Environment Scale: Con- 
struct Definition, Measurement, and Validation. Educational and Psychological Measurement, 64, 165-184. https://doi.org/10.1177/0013164403258440

Swan, M. (2009). Emerging Patient-Driven Health Care Models: An Examination of Health Social Networks, Consumer Personalized Medicine and Quantified Self-Tracking. International Journal of Environmental Research and Public Health, 6, 492-525.

Topol, E. J. (2013). The Creative Destruction of Medicine. New York: Basic Books.

Van Berkel, N., Luo, C., Ferreira, D. et al (2015). The Curse of Quantified-Self: An Endless Quest for Answers. In Proceedings of the 2015 ACM International Joint Conference on Pervasive and Ubiquitous Computing (pp. 973-978). Osaka: ACM. https://doi.org/10.1145/2800835.2800946

Woltin, K. A., \& Yzerbyt, V. (2015). Regulatory Focus in Predictions about Others. Personality and Social Psychology Bulletin, 41, 379-39. https://doi.org/10.1177/0146167214566188

Yao, K. (2008). Review of Self-Efficacy: A New Trend in Organizational Behavior. Journal of Management, 5, 463.

Yin, M., \& Li, Q. (2017). Research on the Willingness to Use Mobile APP Continuously Integrating ECT and IS Success Theory-A Case Study of Health APP. Journal of Dalian University of Technology (Social Science Edition), 38, 81-87.

Zhang, Y. D., \& Li, D. J. (2018). Obstacles to Quantified Self in the Field of Consumption and Their Influencing Mechanism. Progress in Psychological Science, 15, 74-83.

Zhang, Z. T., \& Li, Q. (2015). Are People Who Feel Good about Themselves More Willing to Work Hard? - The Influence of Self-Efficacy on Individuals' Willingness to Work. Economic Management, 37, 134-144.

Zhang, Z., Wang, Y. N., Chen, X. H., \& Gao, Y. (2016). Empirical Study on Factors Influencing MOOC Continuous Learning Intention-Based on Improved Expectation Confirmation Model. Research on Audio-Visual Education, 37, 30-36.

Zhu, G., Ma, L., Sunanda, S. W. et al. (2010). Consumer Adoption Model and Empirical Research Based on Social Cognition Theory. Nankai Management Review, 13, 12-21. 